\title{
Preparedness of a catheterization laboratory for the corona virus (COVID-19) outbreak
}

\section{Short communication}

Coronavirus pandemic that was first reported in Wuhan, China on December $31^{\text {st }} 2019$, took us and the entire world by surprise. We had never dealt with such a pandemic before and we were not prepared in any aspect. Neither in terms of infrastructure, nor with the catheterization laboratory (cath-lab) personnel or equipment. According to current evidence, COVID-19 virus is primarily transmitted through respiratory droplets (i.e. droplet particles $>5-10 \mu \mathrm{m}$ in diameter) and contact routes of infected patients. ${ }^{1-7}$ Fomites in the immediate environment of infected patients is another way of transmission. ${ }^{8}$ Pre-symptomatic transmission i.e. infected persons transmitting COVID-19 during its' incubation period, before symptoms develop had been documented in a small number of case reports and studies. This was the case in truly asymptomatic transmission as well. ${ }^{9-16}$ In the following document, we would like to share with you our local acts and preparations in order to deal with the COVID-19 pandemic crisis in our catheterization laboratory unit.

Abbreviations: STEMI, ST-segment elevation myocardial infarction; NSTEMI, non-ST-segment elevation myocardial infarction; PCI, percutaneous coronary interventions; CDC, center for disease control; HFOT, high flow oxygen therapy; CPAP, continuous positive airway pressure, ETT, endotracheal tube; CPR, cardiopulmonary resuscitation

\section{Introduction}

Our first goal was to prepare the cath-lab infrastructure to deal with an infected scenario. We first organized a meeting with the center's engineer and infectious control experts in order to plan and decide about the steps needed to approach this endeavor. We decided that only the cath-lab room itself will be potentially contaminated and the rest of the unit will remain clean and sterile. For airborne precautions, we had to alter the cath-lab ventilation system to work as a "negative pressure room" i.e. the exhaust flow rate should be greater than the air supplied with all doors and openings closed, and air should be exhausted outside the building via a chimney above the roof, so that it is unlikely to re-enter the building or its ventilation system.

\section{Cath-lab equipment}

The next step was to organize the equipment in the cat-lab. We first evacuated equipment that is not ordinarily required, so that only the most necessary equipment for interventional procedures will remain in the room. We then dedicated a closet that will be regarded as "contaminated", i.e. contain equipment to be used urgently among corona-infected patients, in order to segregate it from the noncontaminated equipment (Figure 1). We also decided to use one brand of catheters, balloons, and stents that contain all sizes and fit most of our cases.

\section{Cath-lab personnel}

We defined that each team will comprise of:

i. Consultant interventional cardiologist
Volume 13 Issue 4 - 2020

\author{
Gabriel Greenberg, Abed Samara, Ran \\ Kornowski \\ Department of Cardiology, Tel Aviv University, Israel
}

Correspondence: Gabriel Greenberg, Department of Cardiology, Tel Aviv University, Ramat Aviv, Israel, Tel +972-52-45 I 8855, Email Gabrigr75@gmail.com

Received: July 08, 2020 | Published: July 21, 2020

ii. Registered nurses - one that will assist directly the patient's management and the operator and the other that will assist the interventional nurse ("circulating nurse"). Both nurses will operate from within the room.

iii. Radiographers - one in the control room operating the imaging equipment there, and the other one in the cath-lab, operating the contrast delivering system, and assisting the rest of the staff, in order to avoid contamination beyond the cath-lab room. The second radiographer eliminates the need to create a lobby (anteroom) and an interlocking door system at the intra-procedural entrance to the cath-lab.

iv. Cardiac physiologist - will operate the monitoring and hemodynamic equipment from within the room as well as other imaging equipment (IVUS/FFR).

Most importantly, we trained all the team members in personal full-body protective equipment donning, usage, and doffing (Figure 2).

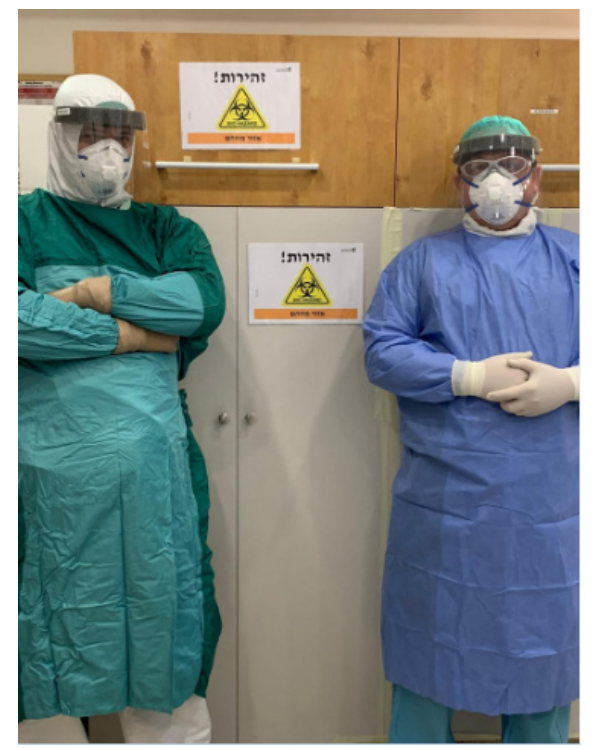

Figure I Contaminated closet. 


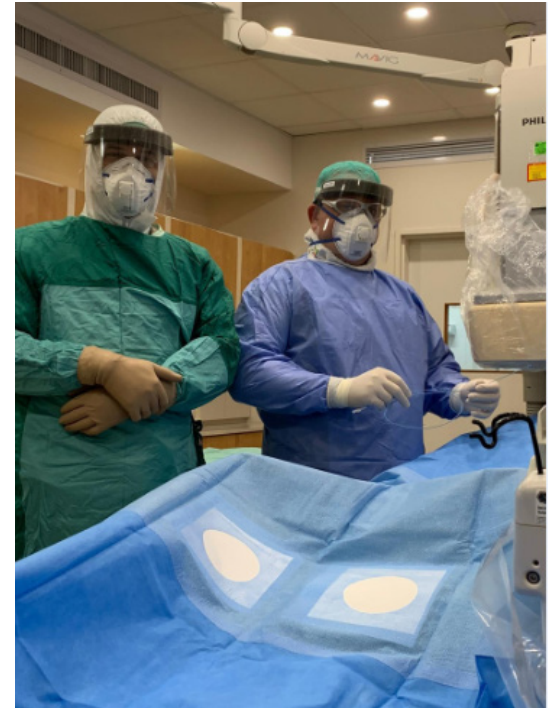

Figure 2 Full Body Personal Protective Equipment.

\section{Patient triage}

Rabin Medical Center is composed of two campuses/ hospitals, one of the campuses had been converted to a "Corona Center", i.e. treating COVID-19 confirmed cases only. The second campus, the "non-Corona center" treats either "Corona suspicious" or "Corona free" cases, i.e. patients who are free of the virus and require treatment for their routine illnesses. The non-Corona center comprises "Respiratory wards" oriented to treat patients with acute respiratory illnesses, COVID-19 suspicious (within negative pressure isolation rooms), and routine acute respiratory illnesses, non-COVID associated. Suspicious cases with laboratory confirmation of virus contraction are transferred to the "Corona Center".

Patients with acute coronary syndromes, i.e. ST-segment elevation myocardial infarction (STEMI), non-ST-segment elevation myocardial infarction (NSTEMI), or unstable angina pectoris that are not suspected of being infected (after thorough anamnesis for symptoms, for contact with possibly infected patients, and travel history) undergo percutaneous coronary interventions (PCI) according to guidelines in the "non-Corona center". For the rest of the patients we decided to adopt the protocol suggested by Ehtisham Mahmud in SCAI's document published on March $16^{\text {th }} 2020$ with mild adjustments.

\section{Confirmed COVID-I 9 patients}

In the presence of STEMI or NSTEMI with ongoing ischemic symptoms/hemodynamic compromise, patients should be transferred to the "Corona center" and should be taken to the Cath Lab for angiography/primary PCI with appropriate infectious disease protection for the entire Cath Lab team. For NSTEMI patients who are otherwise stable and/or at lower risk, to minimize the risk of staff exposure, medical management with coronary angiography for recalcitrant symptoms only is the current approach. Elective coronary angiography in the "non-Corona center" can then be pursued at a future time when the patient is cured from the infectious insult (Figure $3 \mathrm{~A})$.

\section{Possible COVID-1 9 patients}

When these patients present with a STEMI, they should first be admitted to the "non-Corona center", they should be treated with primary PCI and appropriate infectious disease protection. If converted to COVID-19 positive they should be transferred to the "Corona center". When presenting with a NSTEMI, they should be admitted to the "non-Corona center", managed medically, and they should await coronary angiography until a negative COVID-19 test has been obtained unless hemodynamically and clinically unstable (e.g. very high risk NSTEMI). Confirmed COVID-19 cases should be transferred to the "Corona center" (Figure 3B). In selected cases, mechanical circulatory support (e.g. ECMO, Impella device) would demand the use of the cath-lab facility in order to assist in severe COVID19 cases. Nonetheless, we believe that every possible effort should be made in order to minimize patient's mobilization and or cath-lab contamination and ECMO devices could be deployed bedside.

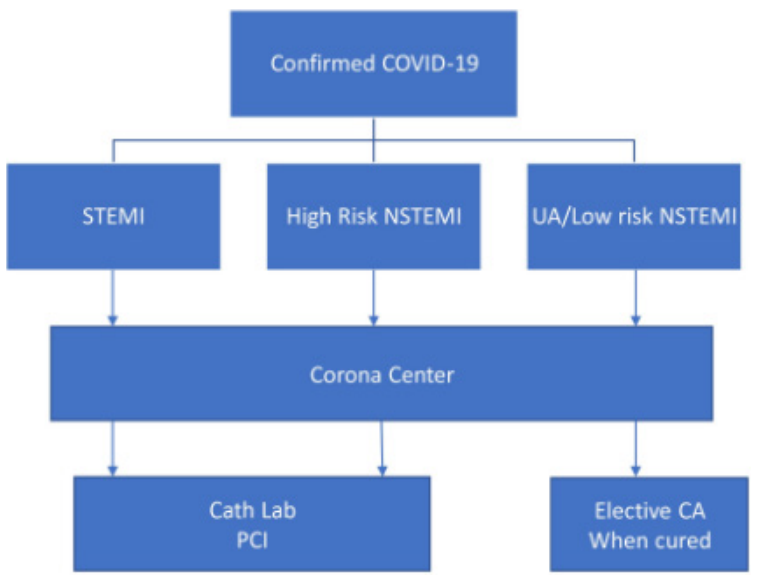

Figure 3(A) Flowchart: Management of confirmed COVID: 19 patients.

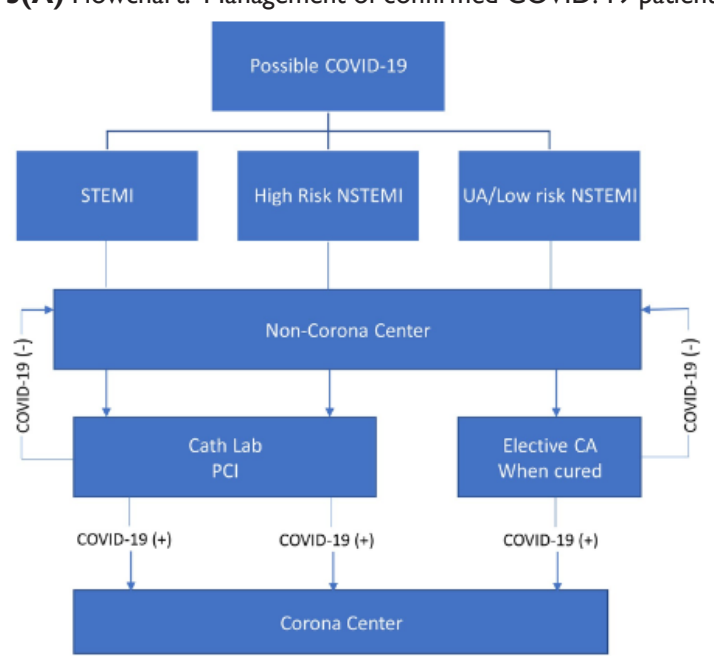

Figure 3(B) Flowchart: Management of possible COVID: 19 patients.

All confirmed COVID-19 patients should be transported to the cath-lab via a stretcher transport isolator (Figure 4), The cath-lab team will wait for them fully- protected. The patient should be moved from the stretcher and transferred to the cath-lab bed only after ascertaining that only the operating team is in the room, the negativepressure system is functioning and the doors are closed.

At the end of the procedure, the patient will be transferred back to the stretcher transport isolator. After ascertaining that it is sealed, team members will wait with the patient in the room for approximately 10 more minutes required for the infected particles to be evacuated out of the room via the ventilation system (agreed upon with the engineer). 
Only then will the doors are to be allowed to open and the patient will be transported back to the ward. At the end of the procedure and after the patient is transferred back to the ward, the cath-lab and its' gear should be cleaned by a dedicated "Commando unit" that was specifically trained, using chlorine solutions at dilution of 2,000 parts per million. Complementary disinfection should be performed by using ultra-violet disinfection robot (Figure 5).

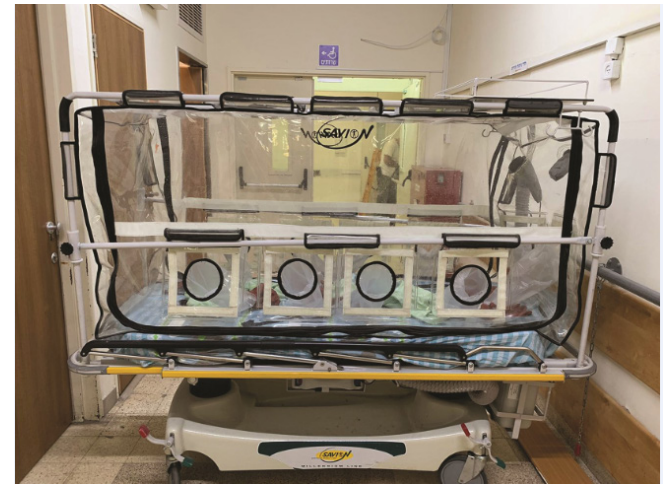

Figure 4 Stretcher transport isolator.

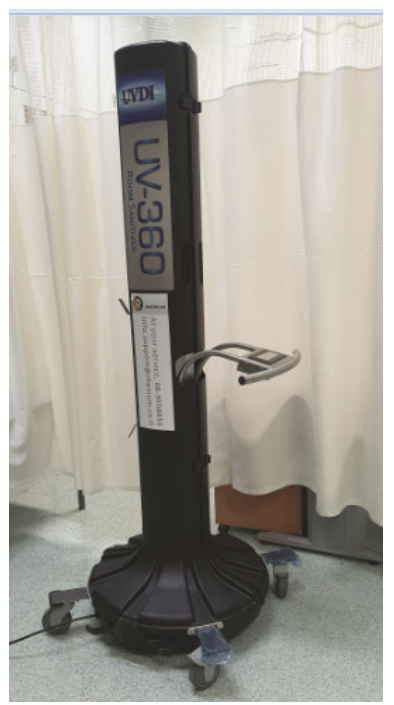

Figure 5 UV disinfection robot.

\section{Semi-elective cath lab patients}

Patients who underwent percutaneous coronary intervention in an elective, urgent or emergent setting and remain with significant residual coronary artery disease that requires complementary revascularization. These patients are invited electively directly to the cath-lab in the "Non-Corona" center and undergo complementary procedures and are discharged the following day.

\section{Elective cath lab patients}

This group of patients requires an approach that is evolving. If social distancing and isolation of the high-risk cohort is to be effective, delaying these elective procedures seems the most prudent approach. The only patients who should be treated are those with accelerating symptoms or those felt to be too unstable for deferral. Structural and valvular interventions should be performed or deferred, based upon the cath-lab readiness, the number of rooms available for interventions in the unit, the ability to isolate those patients from COVID19 environment, the overall COVID19 workload in a specific hospital and the availability of experienced cath-lab and cardiology ward personal to treat those patients.

\section{Outpatient clinic}

Patients who underwent coronary angiography or interventions who require further follow-up and / or work-up are served via telemedicine visits. Information regarding patient's medical history, medications, blood- tests, imaging, is available for the physicians who can respond accordingly. Patients with urgent symptoms/high risk features are invited to our outpatient clinic in the "non- Corona" center following thorough anamnesis and rule-out of acute infectious illness or contact with confirmed Covid-19 patients. Both patients and physicians wear surgical face-masks during contact, if patients' condition requires monitoring and expedited work-up in an in-patient setting, they are admitted and undergo guideline-based medical therapy.

Strategies aimed to minimize disease contraction during high risk periods of transmission as virus transmission occurs primarily via aerosols and fomites, the highest risk periods of transmission occurs during donning and doffing and during airway management. We adopted practices aimed at minimizing this risk.

\section{Donning and doffing}

Since donning is achieved in an entirely "clean" environment and involves an entirely clean gear, the doffing period is the period known to be the most susceptible for contamination, thus particular attention is assigned for that process. Doffing involves two staff members, one that intends to doff and an assistant that assists in that process. Donning and doffing is executed in a sequence suggested by the Center for Disease Control and Prevention (CDC). https://www.cdc. gov/hai/pdfs/ppe/ppe-sequence.pdf

\section{High flow oxygen therapy (HFOT)}

A form of respiratory support used in patients with hypoxemic respiratory failure (mild ARDS), utilizing compressed air and humidification that is delivered at high flow rates via a nasal cannula and aims to reduce the work of breathing. This type of respiratory support is used cautiously as a result of its' potential for aerosolization of viral particles and a heightened risk for contamination of healthcare workers. Thus it is used only in negative pressure rooms and when the staff members are fully protected.

\section{Continuous positive airway pressure (CPAP)/Bilevel positive airway pressure (BiPAP)}

Non-invasive ventilation modalities that support breathing by delivering mechanically assisted breaths via face masks that reduce the effort of breathing without the need for intubation or surgical airway. These modalities are seldomly used in our institution in COVID 19 patients because of the risk of aerosolization in addition to the concerns that these patients can abruptly deteriorate without any warning. Although there is no good evidence to support our approach, in our view early intubation is the preferred approach.

\section{Emergent intubation}

Carried out by the most experienced airway provider, the attending anesthesiologist assisted by a skilled registered nurse, both should be fully protected. Preoxygenation with $100 \%$ oxygen is achieved via 
facemask (2 hand technique to maintain seal) attached to AMBUbag circuit that includes a high efficiency particulate air (HEPA) filter, capnometer, PEEP valve, ambu bag, reservoir bag and an oxygen source. If possible we avoid ventilation to minimize aerosolization. Intubation performed using rapid sequence induction using a video laryngoscope (GlideScope ${ }^{\circledR} \mathrm{Go}^{\mathrm{TM}}$ ) that facilitates rapid first-pass success and concurrently supporting infection control initiative. After successful intubation and cuff inflation, the endotracheal tube (ETT) is connected to AMBU bag circuit and its' position is confirmed by symmetrical chest rise, "fogging" of ETT and capnography (end-tidal $\mathrm{CO} 2$ - consistent waveform and value after delivering of $>3$ breaths). Auscultation via stethoscope is to be avoided to minimize contact Once ETT position is confirmed, it is connected to the ventilator. HEPA filter is attached to the expiratory limb of the circuit in the path of exhaled gas.

\section{Cardiopulmonary resuscitation (CPR)}

Is an aerosol-generating procedure, hence all staff members should comply with airborne precautions and the procedure should optimally be executed in a negative pressure room. Self-adhesive, dual-function monitor/defibrillation electrode pads are attached to the patient and monitoring and defibrillation is executed by pressing a remote button to avoid close contact with the patient. Intubation with cuffed and secured tube and mechanical ventilation with HEPA filter should be pursued at the earliest feasible opportunity in order to maintain a closed circuit and reduce aerosolization. External mechanical chest compression device (i.e. LUCAS device) is used to reduce personnel exposure and risk of contamination.

In order to minimize transmission from pre-symptomatic/truly asymptomatic patients at the "Non-Corona" Center, all patients are evaluated for COVID-19 as they enter the emergency department. Any physical approach/contact with patients at all times involves wearing surgical face-masks, for both patients and staff members.

\section{Summary and take-home message}

We share herein our preliminary preparation scheme for the COVID19 related cath-lab readiness for various clinical scenarios. We believe that the key for success is the organization, readiness, adaptability and changing the old habits to serve the patients' needs while keeping the healthcare personal well protected from infectivity. This scheme is obviously a "moving target" and growing experience collectively will teach us how to best manage and practice interventional cardiology in the COVID19 era.

\section{Acknowledgments}

None.

\section{Conflicts of interest}

Author declares that there are no conflicts of interest.

\section{Funding}

None.

\section{References}

1. World Health Organization (WHO). Modes of transmission of virus causing COVID-19: implications for IPC precaution recommendations. Scientific brief 29 March 2020. Geneva, Switzerland: World Health Organization (WHO); 2020.

2. Liu J, Liao X, Qian S, et al. Community transmission of severe acute respiratory syndrome coronavirus 2, Shenzhen, China, 2020. Emerg Infect Dis. 2020;26(6):1320-1323.

3. Chan J, Yuan S, Kok K, et al. A familial cluster of pneumonia associated with the 2019 novel coronavirus indicating person-to-person transmission: a study of a family cluster. Lancet. 2020;395(10223):514-523.

4. Li Q, Guan X, Wu P, et al. Early transmission dynamics in Wuhan, China, of novel coronavirus-infected pneumonia. $N$ Engl J Med. 2020;382(13):1199-1207.

5. Huang C, Wang Y, Li X, et al. Clinical features of patients infected with 2019 novel coronavirus in Wuhan, China. Lancet. 2020;395(10223):497506.

6. Burke RM, Midgley CM, Dratch A, et al. Active monitoring of persons exposed to patients with confirmed COVID-19 — United States, JanuaryFebruary 2020. MMWR Morb Mortal Wkly Rep. 2020;69(9):245-246.

7. World Health Organization (WHO). Report of the WHO-China Joint Mission on Coronavirus Disease 2019 (COVID-19) 16-24 February 2020. Geneva, Switzerland: World Health Organization (WHO); 2020.

8. Ong SW, Tan YK, Chia PY, et al. Air, surface environmental, and personal protective equipment contamination by severe acute respiratory syndrome coronavirus 2 (SARS-CoV-2) from a symptomatic patient. JAMA. 2020;323(16):1610-1612.

9. World Health Organization (WHO). Coronavirus disease (COVID-19) situation report 73. Geneva, Switzerland: World Health Organization (WHO); 2020.

10. Yu P, Zhu J, Zhang Z, et al. A familial cluster of infection associated with the 2019 novel coronavirus indicating possible person-to-person transmission during the incubation period. J Infect Dis. 2020;221(11):1757-1761.

11. Huang R, Xia J, Chen Y, et al. A family cluster of SARS-CoV-2 infection involving 11 patients in Nanjing, China. Lancet Infect Dis. 2020;20(5):534-535.

12. Pan X, Chen D, Xia Y et al. Asymptomatic cases in a family cluster with SARS-CoV-2 infection. Lancet Infect Dis. 2020;20(4):410-411.

13. Tong ZD, Tang A, Li KF, et al. Potential presymptomatic transmission of SARS-CoV-2, Zhejiang Province, China, 2020. Emerg Infect Dis. 2020;26(5):1052-1054.

14. Wei WE, Li Z, Chiew CJ, et al. Presymptomatic Transmission of SARSCoV-2 - Singapore, January 23-March 16, 2020. MMWR Morb Mortal Wkly Rep. 2020;69(14):411-415.

15. Kimball A, Hatfield KM, Arons M, et al. Asymptomatic and Presymptomatic SARS-CoV-2 Infections in Residents of a Long-Term Care Skilled Nursing Facility — King County, Washington, March 2020. MMWR Morb Mortal Wkly Rep .2020;69(13):377-381.

16. Arons MM, Hatfield KM, Reddy SC, et al. Presymptomatic SARS-CoV2 infections and transmission in a skilled nursing facility. $N$ Engl $\mathrm{J} \mathrm{Med}$. 2020;382(22):2081-2090. 